\title{
Seasonal co-occurrence patterns of bacterial and eukaryotic phytoplankton communities and ecological effects of keystone taxa in an urban river
}

\author{
Jing Yang ${ }^{1}$, Hai-guang Pei ${ }^{1}$, Jun-ping Lv ${ }^{1}$, Qi Liu ${ }^{1}$, Fang-ru Nan ${ }^{1}$, Xu-dong Liu ${ }^{1}$, Shu-lian \\ $\mathrm{Xie}^{1}$, and Jia Feng ${ }^{1}$ \\ ${ }^{1}$ Shanxi University
}

July 29, 2020

\begin{abstract}
Microorganisms play a key role in aquatic ecosystems. Recent studies have showed that some keystone taxa in microbial communities can drive the changes in community composition and function. However, most studies have focused on abundant taxa, whereas rare taxa are neglected because of their low abundance. Therefore, it is important to clarify the seasonal variation of bacterial and microalgal communities and understand the synergistic adaptation of these organisms to different environmental factors. We investigated the bacterial and eukaryotic phytoplankton communities and their seasonal co-occurrence patterns using16S and 18S rDNA sequencing approach. Our results indicated that in eukaryotic phytoplankton networks, spring and autumn networks had higher connectivity and complexity, forming the highly stable community structure. The positive interactions of bacterial network were significantly higher than the negative interactions, indicating that more mutual cooperation can make the microbial communities better resist changes in the external environment, thereby maintaining the stability of microbial network. The main genera identified as keystone taxa in bacterial networks were Pseudomonas, Stenotrophobacter, Bosea, and Hyphomicrobium, which were significantly related to many predicted functions. The main genera identified as keystone taxa in eukaryotic phytoplankton networks were Monodus, Tetradesmus, Scenedesmus, Monoraphidium, and Amphora, which were affected by dissolved organic carbon, nitrate, nitrite, and phosphate, changes in these environmental factors can affect the stability of network. Through the co-occurrence patterns, we analyzed the internal mechanism of interaction between bacteria and eukaryotic phytoplankton and understood the potential importance of keystone taxa in ecological processes such as carbon, nitrogen, and phosphorus dynamics.
\end{abstract}

Seasonal co-occurrence patterns of bacterial and eukaryotic phytoplankton communities and ecological effects of keystone taxa in an urban river

Jing Yang · Haiguang Pei · Junping Lv · Qi Liu · Fangru Nan · Xudong Liu · Shulian Xie and Jia Feng

School of Life Science, Shanxi University, Taiyuan 030006, China

Corresponding author: Jia Feng fengj@sxu.edu.cn

Running title: Co-occurrence of microbiota and environment

\begin{abstract}
:
Microorganisms play a key role in aquatic ecosystems. Recent studies have showed that some keystone taxa in microbial communities can drive the changes in community composition and function. However, most studies have focused on abundant taxa, whereas rare taxa are neglected because of their low abundance. Therefore, it is important to clarify the seasonal variation of bacterial and microalgal communities and understand the synergistic adaptation of these organisms to different environmental factors. We investigated
\end{abstract}


the bacterial and eukaryotic phytoplankton communities and their seasonal co-occurrence patterns using $16 \mathrm{~S}$ and $18 \mathrm{~S}$ rDNA sequencing approach. Our results indicated that in eukaryotic phytoplankton networks, spring and autumn networks had higher connectivity and complexity, forming the highly stable community structure. The positive interactions of bacterial network were significantly higher than the negative interactions, indicating that more mutual cooperation can make the microbial communities better resist changes in the external environment, thereby maintaining the stability of microbial network. The main genera identified as keystone taxa in bacterial networks werePseudomonas , Stenotrophobacter , Bosea, andHyphomicrobium , which were significantly related to many predicted functions. The main genera identified as keystone taxa in eukaryotic phytoplankton networks were Monodus, Tetradesmus, Scenedesmus, Monoraphidium, and Amphora, which were affected by dissolved organic carbon, nitrate, nitrite, and phosphate, changes in these environmental factors can affect the stability of network. Through the co-occurrence patterns, we analyzed the internal mechanism of interaction between bacteria and eukaryotic phytoplankton and understood the potential importance of keystone taxa in ecological processes such as carbon, nitrogen, and phosphorus dynamics.

Keywords:Seasonal co-occurrence patterns; Bacteria and eukaryotic phytoplankton communities; Keystone taxa; Ecological effect; Urban river

\section{Introduction}

Urban rivers and lakes are important to human culture, welfare, and development, but they have suffered from long-term serious pollution because of the various anthropogenic activities and discharge of untreated or coarsely treated wastewater from sewage treatment plants (Gücker et al., 2011). These pose a threat to the health of aquatic ecosystems and can change the physio-chemical environment and microbial community composition (Bradley, Emma, \& Kelly, 2013). Pervious researches have demonstrated that the effluent discharge can lead to high nutrients pollution (nitrate, phosphate, and ammonium) and organic matter pollution (permanganate index, chemical oxygen demand, and suspended solids), which has an adverse effect on the functions of ecological community and aquatic ecosystems (Gücker, Brauns, \& Pusch, 2009; Waiser, Tumber, \& Holm, 2011). As an important part of urban river ecosystems, microbial communities play an irreplaceable role in participating in the biogeochemical processes and nutrient cycling (Azam, 1998; Lawrence, Swerhone, Wassenaar, \& Neu, 2005) and are the ideal variables for monitoring the ecological impact of anthropogenic activities on the functional characteristics in river water environment.

In natural ecosystems, individual organisms do not exist in isolation, but coexist with each other to form a network of ecological interactions. These complex associations mediate the influence of biodiversity on ecosystem functions (Duffy, Cardinale, Mcintyre, Thebault, \& Loreau, 2007). Microorganisms (such as, bacteria and microbial eukaryotes) are the important contributors to the biodiversity and can play important roles in the food web and biogeochemical cycle (Azam \& Malfatti, 2007; Buchan, Lecleir, Gulvik, \& Gonzalez, 2014). Microbial community structure is not only affected by interactions between species, but also by physicochemical parameters and regional conditions (Jones et al., 2013; Yannarell \& Triplett, 2005). The co-occurrence patterns revealed based on metagenomic sequencing can predict the positive and negative ecological interactions between environmental variables and various species in aquatic ecosystems (Bunse et al., 2016). Network analysis can determine the keystone species that are necessary to maintain community stability among many species. Keystone species can affect the entire microbial community through a series of pathways. For example, they can affect the community structure and function through the adjustment of some intermediate groups and effect groups. Besides, keystone species also susceptible to dynamic environment, the disappearance or change of keystone species may cause disturbance to mature communities (Steele et al., 2011).

The microbial communities are composed of abundant taxa with high abundance and rare taxa with low abundance. Among them, the types of abundant taxa are few, and most of them are rare taxa (Logares et al., 2013). Abundant taxa are conductive to the organic matter flux and biomass yield (Pedrosalio, 2012), so the study of their community diversity is of great significance for deeply understanding ecological function. The emergence of rare species is due to an unfavorable environment, but when the environment becomes 
favorable, its abundance increases. Studies have shown that rare taxa can accelerate the degradation of organic matter, which is of great significance to the nutrition cycle (Pester, Bittner, Deevong, Wagner, \& Loy, 2010). Indigenous rare taxa in environment can control the invasion of foreign microorganisms (such as certain foreign pathogens) (Mallon et al., 2015), these results indicated that rare microorganisms also play an irreplaceable role in the ecosystem. However, due to the limitation of methods, the previous community analysis mainly focused on relatively rich taxa through microscopic observation (Yu et al., 2014). Metagenomics are the research of collective microbial genomes recovered directly from environmental samples, which is independent on culture or prior knowledge of microbial communities (Rastogi \& Sani, 2011). Metagenomics investigations have been performed in various environments (e.g., the ocean, soil, phyllosphere, and sediment), can provide the functional diversity and phylogenetic of uncultured microorganisms and identify the rare taxa (Jo, 2004).

The trend of microbial succession in time has been fully confirmed, but the formation mechanism of microbial temporal succession is still unclear. The main purpose of this study is to understand the formation and maintenance mechanism of microbial diversity (including abundance and composition). On this issue, the most mainstream views are microbial community construction is subject to a combination of deterministic processes (based on niche theory, including species interactions, environmental selection, etc.) and stochastic processes (based on neutral theory, including diffusion limitation, drift, etc.) (Stegen, Lin, Konopka, \& Fredrickson, 2012). The complex interrelationship networks can reflect the inherent mechanism of microbial interaction on environmental interference (Hunt \& Ward, 2015). On this basis, we could also explore the topological characteristics of these networks and identify the keystone species based on statistical analysis. Although a great deal of studies has been conducted on the interactions between abundant taxa (Chen, Chen, Xing, Li, \& Wu, 2010; Ger et al., 2016), few studies has investigated how rare bacterial taxa and eukaryotic phytoplankton taxa affect the community structure of microorganisms in aquatic ecosystem. So far, cooccurrence patterns of rare taxa in urban rivers still has little attention. Therefore, identified key species in our study area were divided into abundant taxa and rare taxa. In this study, metagenome sequencing based on $16 \mathrm{~S}$ and 18S rDNA genes were used to detect the seasonal pattern of bacterial and eukaryotic phytoplankton communities in an urban river, network analysis was used to identify keystone species necessary to maintain community stability, and discussed the relationship between these keystone species and ecological functions and environmental variables. Our research will help to understand the interactions and ecological functions of microorganisms in depth, and improve the ability to predict the response of microorganisms to environmental changes.

\section{Materials and methods}

\section{Sampling and environmental parameters}

The study area was located in the Fenhe River of Shanxi province, northern China (latitude $35^{\circ}-39^{\circ} \mathrm{N}$; longitude $110^{\circ}-114^{\circ} \mathrm{E}$ ) (Fig. 1). We collected 144 samples from January 2019 to December 2019 at 6 sampling stations. A $2 \mathrm{~L}$ sampler was used to collect water samples at the depth of $0.5 \mathrm{~m}$, then filtered through 0.22 $\mu \mathrm{m}$ mixed fiber microporous membranes. And the fraction $>0.22 \mu \mathrm{m}$ was used for DNA extraction, the corresponding water samples were used to determine the physical and chemical parameters. The nitrate $\left(\mathrm{NO}_{3}{ }^{-}\right)$, nitrite $\left(\mathrm{NO}_{2}{ }^{-}\right)$, and phosphate $\left(\mathrm{PO}_{4}{ }^{3-}\right)$ were measured following the methods in Wang, Lou, Sun, and Song (2008). The dissolved organic carbon (DOC) concentration was determined according to the method described by Bolan, Baskaran, and Thiagarajan (1996). The Water and Wastewater Monitoring Analysis Method was used to measure Chlorophyll a (Chl a) concentrations (Zhu et al., 2019).

\section{DNA Extraction, PCR Amplification, and Products Purification}

Genomic DNA was extracted from environmental samples using E.Z.N.A ${ }^{\text {TM }}$ Mag-Bind DNA Kit (OMEGA) as per manufacturer's instructions. Agarose gel was used to detect the purity and concentration of DNA extracts, and then selected the qualified samples for subsequent analysis. The V3-V4 region of 16S rDNA gene was amplified using primers 341F (5'-CCTACGGGNGGCWGCAG-3') and 805R (5'GACTACHVGGGTATCTAATCC-3') (Herlemann et al., 2011); the V4 region of 18S rDNA gene was ampli- 
fied using primers V4F (5'-GGCAAGTCTGGTGCCAG-3') and V4R (5'-ACGGTATCTRATCRTCTTCG3') (Sun et al., 2015). The PCR amplification cycle was: the first round of amplification was $94^{\circ} \mathrm{C}$ for $3 \mathrm{~min}$, followed by 5 cycles at $94^{\circ} \mathrm{C}$ for $30 \mathrm{~s}, 45^{\circ} \mathrm{C}$ for $20 \mathrm{~s}, 65^{\circ} \mathrm{C}$ for $30 \mathrm{~s}$, then followed by 20 cycles at $94^{\circ} \mathrm{C}$ for 20 $\mathrm{s}, 55^{\circ} \mathrm{C}$ for $20 \mathrm{~s}, 72^{\circ} \mathrm{C}$ for $30 \mathrm{~s}$, and a final extension at $72^{\circ} \mathrm{C}$ for $5 \mathrm{~min}$; the second round of amplification was $95^{\circ} \mathrm{C}$ for $3 \mathrm{~min}$, followed by 5 cycles at $94^{\circ} \mathrm{C}$ for $20 \mathrm{~s}, 55^{\circ} \mathrm{C}$ for $20 \mathrm{~s}, 72^{\circ} \mathrm{C}$ for $30 \mathrm{~s}$, and a final extension at $72^{\circ} \mathrm{C}$ for $5 \mathrm{~min}$. Two round PCR reactions were carried out in $30 \mu \mathrm{L}$ reactions, including $15 \mu \mathrm{L} 2 \times$ Taq master Mix, $10 \mu \mathrm{M}$ of forward and reverse primers, and $20 \mathrm{ng}$ genomic DNA or PCR products of first round amplification, respectively.

\section{Sequencing and Data Analysis}

The gene amplicons were sequenced on the Illumina Miseq platform, effective tag readings were gained by trimming the terminal bases, removing primer sequences and barcodes, and filtering the low-quality bases. The non-specific amplified sequences were removed using Usearch, chimeras were detected by Uchime algorithm (version 4.2.40) (Edgar, Haas, Clemente, Christopher, \& Rob, 2011). Subsequently, we then blastn aligned the representative sequence in the database with the chimeric-removed sequence. Alignment results below the threshold were considered to be the sequence outside the target region and eliminated this part of the sequence (Haas et al., 2011). Then using the open reference OTU to cluster the qualified sequences into the species-level OTU (Operational Taxonomic Units), and the similarity cut-off value of using UCLUST in QIIME was 97\%. The classification of rare and abundant species relies on the cutoff level of relative abundance, set rare taxa to 0.1 or $0.01 \%$ and abundant taxa to $1 \%$ (Zhang et al., 2018). In our study, we classified OTUs with a relative abundance greater than $1 \%$ as abundant taxa and less than $0.01 \%$ as rare taxa. In order to gain the species classification information corresponding to each OTU, the OTU representative sequence was aligned with the Silva database using the RDP Classifier algorithm with an $80 \%$ confidence threshold, and annotate species information at various levels (Wang, Garrity, Tiedje, \& Cole, 2007). All raw sequences data in this study were deposited into the NCBI Sequence Read Archive (SRA) database under the accession number of SRP273360.

The co-occurrence network of each season was established through Molecular Ecological Network Analysis (MENA) based on the relative abundance of OTUs (Deng et al., 2012; Zhou et al., 2010), which computed the Pearson Correlation Coefficients (PCC) for each OTU pair, and then calculated the statistical significance of PCC values by a permutation test. The similarity threshold of microbial communities was determined according to the method of random matrix theory. And the correlation is significant $(P<0.01)$ when the correlation between the two OTUs is greater than the similarity threshold. Edges were set between pairs of OTUs which the PCC was significant. Then, the networks were visualized with Gephi and Cytoscape (Assenov, Ramirez, Schelhorn, Lengauer, \& Albrecht, 2008). At the same time, in order to compare the difference between the molecular ecological network and the corresponding random network, the topological properties based on Erdós-Réyni random network were calculated using R language.

Then, we used the greedy modularity optimization method to characterize the network modularity of each network established in each season (Shi et al., 2016). Modules greater than 0.4 were used as the threshold of the detection module. In order to determine the topological properties of each node in the network, the nodes were divided four categories through their among-module connectivity $(P i)$ and within-module connectivity $(Z i)$ : Network hubs $(Z i>2.4$ and $P i>0.6)$, Module hubs $(Z i>2.5$ and $P i<0.6)$, Connectors $(Z i<2.5$ and $P i>0.6$ ), and Peripherals $(Z i<2.5$ and $P i>0.6)$ (Deng et al., 2012; Zhou et al., 2010).

PICRUSt is a bioinformatics tool that predicts metagenome gene functional content using $16 \mathrm{~S}$ rDNA. PICRUSt uses the existing gene content annotations in the Integrated Microbial Genome (IMG) database (Markowitz et al., 2012) v3.5 (2,590 genomes) as well as the 16S copy number and functional classification scheme of the reference microbial genome to classify the expected metagenome content. And the current PICRUSt software (version 1.1.0) supports two types of functional prediction. We applied the more popular KEGG Orthologs in this study (Kanehisa, Goto, Sato, Furumichi, \& Tanabe, 2012) for subsequent analysis.

\section{Results}




\section{Physical, chemical, and biotic characteristics of water quality}

The nutrient concentrations, physical properties, and Chl a concentration in temporal were shown in Table 1. The lowest values of nitrate, nitrite, phosphate, $\mathrm{Chl}$ a, and DOC were detected in winter. The concentrations of nitrate in spring were generally higher than in other seasons. The nitrite and phosphate concentrations in summer ranged from $0.083-0.170 \mathrm{mg} \mathrm{L}^{-1}, 0.128-0.212 \mathrm{mg} \mathrm{L}^{-1}$, respectively, which were also higher than those in other seasons. The level of $\mathrm{Chl}$ a in autumn was far in excess of the other seasons. The maximum of average DOC concentration was detected in spring.

\section{Seasonal succession of bacterial and eukaryotic phytoplankton communities}

The bacterial community composition at the genera level was shown in Fig. 2. Our results demonstrated that Proteobacteria (12.35-92.4\%) was the most abundant bacterial phyla, followed by Firmicutes (0.98.19\%), Bacteroidetes (2.83-7.11\%), Planctomycetes (0.94-5.27\%), Actinobacteria (0.49-4.64\%), Verrucomicrobia (0.14-1.78\%), and Cyanobacteria (0.04-2.1\%). Cyanobacteria were represented by Synechocystis andPlanktothrix . At higher taxonomic resolution, the most enriched genus based on relative abundance include Comamonas ,Straphylococcus, Sphingomonas, Acinetobacter, Brevundimonas, Vogesella, Citrobacter , and Clostridium in spring; Acinetobacter ,Chryseobacterium, Comamonas, Exiguobacterium ,Pseudomonas, and Sphingomona in summer;Acinetobacter, GpIIa, Chryseobacterium, Pseudomonas, Comamonas , Stenotrophomonas ,Flavobacterium , Exiguobacterium, and Sphingobium in autumn; Pseudomonas, Flavobacterium, Blastomonas, Undibacterium, Clostridium, and Janthinobacteriumin winter.

The variations in eukaryotic phytoplankton community composition at the genera level were shown in Fig. 3 . The identified sequences were assigned that eukaryotic phytoplankton phylum was composed of Chlorophyta (75.4-93.67\%), Bacillariophyta (4.13-17.23\%), and Ochrophyta (0.19-43.27\%). Results demonstrated that Chlorophyta were the major dominate phyla in Fenhe River. The peak of Ochrophyta occurred in winter. Desmodesmus was the dominant genus in the phylum of Chlorophyta, followed by Mychonastes ,Nephrochlamys , Coelastrum, Discostella, and Chlorella, and in the phylum of Bacillariophyta Cyclotellawas the main genus in spring. Mychonastes, Desmodesmus ,Pseudopediastrum, Nephrochlamys, and Golenkiniawere the most enriched genus in summer. The community was characterized by green algae Mychonastes , Scenedesmus ,Polyedriopsis, Neodesmus, and diatoms Discostellain autumn. The dominant genera were Monoraphidium ,Parietochloris , Chlorella, Nannochloropsis, andLessonia in winter.

\section{Co-occurrence networks ofbacterial communities}

The interaction between different plankton plays a vital role in shaping the distribution pattern of microorganisms. They do not exist in the natural environment in isolation, but form a complex ecological interaction network. Aquatic ecosystems are composed of networks connecting different organisms of different sizes, and are maintained through species-to-species interactions (including symbiosis, competition, and parasitism) (Zhu, Hong, Zada, Hu, \& Wang, 2018). Therefore, co-occurrence patterns of each season were established to explore the succession of OTU associations, which could not only reflect the potential interactions between microbes in complex communities, but also reflect ecological processes such as historical effects, cooperation, and habitat filtering.

The topological properties of bacterial networks over time were presented in Table S1. Overall, the bacterial network in spring was larger and more complex (Fig. 4). The proportion of positive interactions in spring, summer, and winter accounted for $100 \%$. In the co-occurrence networks, the positive interaction was mainly considered as cooperation (Ju, Xia, Guo, Wang, \& Zhang, 2014). Although in the autumn network, in addition to the positive interactions accounted for $82 \%$, the negative interactions also accounted for $18 \%$. However, the numbers of positively correlated connections between nodes were always higher than the negatively correlated connections, indicating that more mutual cooperation can make the microbial communities better resistant to changes in the external environment, thereby maintaining the stability of microbial network (Xue et al., 2018). Compare some network topology parameters of empirical network and the Erdös-Réyni random network, including the modularity index, the average path length, and the average clustering coefficient. The results demonstrated that the parameters of the empirical network were significantly higher than random 
network, which reflected that the network structure was not randomly distributed. The modularity of all networks was higher than 0.4, demonstrating that networks had the modular structure composed of closely connected nodes and forming a "small world" topology.

Among the nodes of the bacterial networks, Proteobacteria occupied the largest number of OTUs in all seasons. And Proteobacteria occurred in almost all of the sub-network regardless of season (Fig. 4). These results demonstrated that some Proteobacteria members can adapt to different ecological environments, and this adaptation could also explain the great abundance of Proteobacteria in all sites in each season. Therefore, bacteria of Proteobacteria always maintained a higher abundance compared with other phyla. The network is divided into Network hubs, Module hubs, Connectors, and Peripherals according to the among-module connectivity $(\mathrm{Pi})$ and within-module connectivity $(\mathrm{Zi})$. OTUs that are divided into Network hubs, Module hubs, and Connectors are regarded as keystone species (Fig. S1 \& S2). These nodes can keep the network at the lowest hierarchical structure under the premise of completing its ecological functions (Fan, Weisenhorn, Gilbert, \& Chu, 2018).

Networks hubs were only detected in spring and autumn. In the spring network, one Network hubs identified originated from Proteobacteria (Pseudomonas ); two Module hubs belonging to Planctomycetes (Pirellula ) and Proteobacteria (Sphingomonas ); five OTUs were divided into Connectors, which were two Proteobacteria OTUs, two Bacteroidetes OTUs, and one Planctomycetes OTU (Gemmata), respectively. In the summer network, one OTU was divided into Module hubs, belonging to Verrucomicrobia at the phylum level; six OTUs were classified as Connectors, the genera that could be identified as keystone taxa were mainly Luteimonas, Gemmata,Mycobacterium, Stenotrophobacter, and Sphingopyxis . In the autumn network, the Bosea genus was divided into Network hubs; two out of three Module hubs were Proteobacteria (Agrobacterium and Hyphomicrobium) and one was Bacteroidetes (Chryseobacterium ); four OTUs were classified as Connectors, genera that could be identified as key taxa includedPseudomonas , Acidovarax , and Rhodoplanes. In the winter network, three Module hubs identified originated from Proteobacteria and Planctomycetes; two Connectors belonging to Verrucomicrobia and Proteobacteria at the phylum level.

\section{Co-occurrence networks of eukaryotic phytoplankton communities}

In the network topological properties of eukaryotic phytoplankton, the average path coefficient, average path length, and modularity index in the molecular ecological network were greater than the corresponding values of the random network, indicating that the network constructed in this study conformed to the characteristics of scale-free, small world, and modular, which can be used for the subsequent study of phytoplankton interaction. The number of nodes and connections in the spring network was significantly higher than in other seasons, with a larger and more complex network, followed by autumn (Fig. 5). The greater complexity of the microbial network usually contributes to the stability of the microbial communities. It can be seen that high and low temperatures are not conducive to the stability of eukaryotic phytoplankton communities. In summer and autumn, the network modularity was relatively higher, and the connections between microorganisms were closer. In the summer and winter networks, positive correlation accounted for $47 \%$ and $43 \%$, negative correlation accounted for $53 \%$ and $57 \%$, respectively. On the contrary, in spring and autumn, the algal communities almost completely tended to co-express, that is, the positive relationship accounted for a higher proportion (spring: 100\%, autumn: 94\%). In the spring network, the Module hubs identified originated from Neodesmus ; Connectors between network came from Ochrophyta (Monodus ) and Chlorophyta (Tetradesmus ). In the summer network, Desmodesmus genus was divided into Network hubs; two OTUs were divided into the Module hubs, both of which were Chlorophyta, but only appraisal forMychonastes ; twelve Connectors were identified as keystone taxa were mainly Dangeardinia, Chlamydomonas ,Lagerheimia, Choricystis, Monoraphidium, andTetradesmus. In the autumn network, two Module hubs were Ochrophyta (Monodus ) and Chlorophyta; five Connectors mainly identified originated from Scenedesmus, Characiopsis, and Cyclotella. In the winter network, one Bacillariophyta OTU (Amphora) was divided into Network hubs; nine OTUs were classified as Connectors, all of which were Chlorophyta at the phylum level, the identified genera including Pseudomuriella,Messastrum, Mychonastes, and Monoraphidium .

\section{Discussion}


Identifying the interactions between bacteria and microeukaryotes and the combination of complex ecological communities is critical for the management of biodiversity, especially in areas with strong human interference and fragile ecosystems. OTUs belonging to keystone taxa are nodes with the low betweenness centrality values and high degree, which are very significant for maintaining the stability of the ecosystem (Ma et al., 2016). Regarding microbial communities, several hypotheses have been proposed, including "neutral theory" and "niche theory" (Dumbrell, Nelson, Helgason, Dytham, \& Fitter, 2010). The Neutral theory predicts that community structure and composition are relevant to the geographic distance between samples, which is due to the dispersal restrictions, because many species have equal functions in the ability to utilize niche. The niche theoretical predicts that changes in species community composition are related to variations in environmental parameters (Jongman, Ter Braak, \& Van Tongeren, 1995), because species have unique characteristics that enable them to develop unique habitats that can be used. Here, we pointed out the functional roles and environmental variables associated with keystone species. By looking for related changes in the abundance of natural taxa and ecosystem functions, we can infer which processes are important.

\section{Relationship between the keystone species and functional roles}

All keystone taxa in the bacterial co-occurrence networks belonging to the rare species (Table S2), indicating that rare taxa play a critical role in the maintenance of microbial community structure. And we found that the positive interactions between rare species and non-rare species were greater than negative interactions. This interactions between species can provide the function and stability of ecosystems, for example, the rare Symbiodinium taxa can significantly improve in the stability of host-symbiont communities in a changing environment (Ziegler, Eguiluz, Duarte, \& Voolstra, 2018). Besides, the cooperation among these taxa also can contribution to the adaptability of microbial communities in complex environments, because the interaction networks can offer a buffer for resisting the environmental disturbance (Konopka, Lindemann, \& Fredrickson, 2015). On the other hand, recent article reported that low-abundance taxa could function as keystone species in the rhizosphere networks (Shi et al., 2016), the disappearance or change of these keystone species can lead to the disintegration of modules and networks, even sub-network (Guimera \& Amaral, 2005). And rare microorganisms maintain a huge functional gene library, which can indirectly enhance the function of abundant microorganisms (Jousset et al., 2017). Therefore, rare taxa are equal to or more important than the abundant taxa in sustaining the stability of ecosystems. The microbial communities have a rich diversity of species. Each species has its own set of genes, cell composition, and metabolic reactions, and it is constantly in complex interaction with the surrounding environment. Based on the correlations between keystone species abundance and functional abundance (Fig. 6), we found that Pseudomonas was negatively correlated with most functions in spring, for example, Membrane Transport, Cellular Processes and Signaling, Immune System, Metabolism of Terpenoids and Polyketides, Xenobiotics Biodegradation and Metabolism, and Excretory System. The keystone species in summer were related to Cardiovascular Diseases, Nervous System, Cell Communication, Sensory System, and Neurodegenerative Diseases. In autumn, Bosea and Hyphomicrobium were grouped together, belonging to Rhizobiales, and participate in many of the same functions, indicating that microorganisms with similar species composition are also similar in functional composition.

The interactions between OTUs within the phylum or high-level taxa (Fig. S3) indicated that taxonomically closely associated bacteria were also ecologically closely related, which in turn also reflected their synergistic relationships or common niche preferences. Apart from the co-occurrence patterns between intra-phylum, such non-random patterns also occurred between different phylum or between different high-level taxa. A typical example was that members of the phyla Proteobacteria were significant correlations with Bacteroidetes, Planctomycetes, and Actinobacteria.

\section{Relationship between the keystone species and environmental variables}

The eukaryotic phytoplankton in the freshwater ecosystem responds rapidly and strongly to environmental disturbances and is considered to be the most important indicators reflecting environmental changes and ecosystem status. Most of the keystone species detected in the eukaryotic phytoplankton co-occurrence patterns were affiliated to the rare taxa, and only a small proportion belong to the abundant taxa (Table S3), indicating that rare taxa are still crucial in the aquatic ecosystem. Therefore, we studied the different and 
complex responses of these keystone species to environmental changes (Fig. 7). We found that DOC, nitrate, and $\mathrm{Chl}$ a were significantly negative related to Monodus. Monoraphidium showed significantly negative correlation with phosphate. Scenedesmus was significantly negative related to nitrate, whereas Amphora was positive correlation with nitrate. As the abundant taxa, Scenedesmus showed a significant positive relationship with DOC.

The less relevant co-occurrence pattern between OTUs within the phylum was observed (Fig. S4), which was consistent with previous observation of taxa over-dispersion in plant or animal natural communities (such as sedge community and mammalian community) (Ju, Xia, Guo, Wang, \& Zhang, 2014). This not only confirmed that over-dispersion of phylogeny may be a common feature of all biological communities from microorganisms to macro plants and animals (Hornerdevine \& Bohannan, 2006), but also showed the important influence of negative interactions (such as competition) on community assembly.

\section{Co-occurrence patterns between bacterial communities and phytoplankton taxa}

In the natural environment, the existence of microorganisms is not isolated from the world. Each microorganism needs to be in contact with the natural environment and other microorganisms during its growth process, thereby form the entire microbial communities. Aquatic ecosystems are heterogeneous environment, which contains enormously diverse prokaryotic and eukaryotic communities. The interactions between prokaryotic and eukaryotic communities also can decide the community structure (Prosser et al., 2007), therefore, we perform the non-random co-occurrence networks and important inter-taxa relationships using network analysis approach. The interactions between phytoplankton and bacteria play a significant role in shaping the microenvironment around these organisms. This microenvironment, known as phycosphere, is thought to shape the diversity of bacteria around phytoplankton, thereby stimulating various interactions between the two groups and affecting the global biogeochemical cycle (Gregory et al., 2018). Interrelations between bacteria and microalgae are multifaceted and complicated, for example, bacteria naturally can rely on photosynthetic phytoplankton to obtain the organic carbon needed to maintain their growth (Falkowski, Fenchel, \& Delong, 2008); in turn, phytoplankton can depend on bacteria to remineralize organic matter into inorganic substitutes, ultimately supporting the growth of algae (Worden et al., 2015). Therefore, the research of phycosphere and bacterial communities is important because they control the metabolic interactions of algal-bacteria in the microenvironment.

TheMychonasteswas distributed worldwide and has a wide range of habitats, including streams and large still waters, for example, Baringo and Victoria Lakes in Kenya, Erken Lake in Sweden, and Stechlin Lake in Germany, etc (Krienitz, Bock, Dadheech, \& Proschold, 2011). However, as the most abundant genus in spring, summer, and autumn in our study,Mychonastes was first reported by Li et al (2013) as a new recorded genus of freshwater Chlorophyceae isolated from Lake Dianchi.Synechococcus is a common prokaryotic picocyanobacterial, which had a relatively high abundance in summer. Liu, Shi, Fan, Wu, and Lei (2019) studied the growth and interspecies competition ofMychonastes and Synechococcus under different $\mathrm{N}: \mathrm{P}$ ratios. Although we did not find a competitive relationship betweenMychonastes and Synechococcus in our results, Mychonastes has a significantly negative correlation with other cyanobacteria (Fig. 8). In Proteobacteria taxa, Maliki a in spring, Brevundimonas andAeromonas in autumn could promote the growth ofDesmodesmus, whileFlavobacterium andMassilia were mutually exclusive with Desmodesmus .Flavobacteriumis considered as a plant growth promoting bacterium, which is reported to exist during the algal blooms and responsible to improve the biological phosphorus removal ability (Park et al., 2007; Pinhassi et al., 2004). In our study, Flavobacterium contributed to the growth of most green algae, Navicula (Bacillariophyta), andEustigmatos (Ochrophyta), while it had the significant negative correlation with Desmodesmus , Pseudomuriella ,Parietochloris, and Goniomonas . Node green algaeLobosphaera had the highest connectivity, with 12 negatively correlated nodes and 4 positively nodes. The stability of ecosystem function lies in the interaction between species (Zhou et al., 2010). Lower connectivity in the community will lead to higher functional stability of the system (for example, a non-scale network), because the entire network module is less affected by node loss (Yang et al., 2017). As an abundant bacterium in autumn,Sphingobacterium was only helpful to the growth of green algae Schroederia, Lee, Oh, Oh, Kim, and Ahn (2016) also reported 
that this bacterium responsible for the growth of algae. Comamonas belonging to Comamonadaceae family had the relatively higher abundance in spring, summer, and autumn, which was considered as denitrifying polyphosphate accumulating microorganism (Calderer et al., 2014).

The negative correlations in co-occurrence patterns may imply predation or competition among taxa. Cyanobacteria are the only prokaryotes with oxygen-producing photosynthesis. They exist in different niches and are important participants in the global carbon and nitrogen cycle. Spring cyanobacteria only had a significant negative correlation withAtlantibacter, and promoted the growth of the remaining bacteria (mainly Firmicutes) and green algae (Golenkinia ). The planktonic cyanobacterium Synechococcus is ubiquitous in the ocean and fresh waters, and plays an important role in total carbon sequestration on a global scale in oligotrophic aquatic environments (Callieri, Cronberg, \& Stockner, 2012). Summer cyanobacteria were more mutually exclusive with Proteobacteria, specifically, Synechococcuswas significantly negative related to Lysobacter. WinterPseudoxanthomonas, Shigella, and Methylobacteriumwere the prevalent (negative) group among theSynechocystis -associated bacteria. Therefore, these results demonstrated that inoculation of functional bacteria can rebuild the microbial communities, which is conducive to the growth of microalgae and inhibits the growth of cyanobacteria.

Numerous evidences have been demonstrated that a link occurs between diatoms and microorganisms (Gregory et al., 2018; Stanish et al., 2013), however, whether they cooperate with each other or exclude each other still lacks relevant knowledge. In our results, we found significant correlations between diatom communities and bacteria communities. And these relationships were greatly subjected to seasonal variations.Discostella had no significant correlation with any bacterial taxa in spring, summer, and winter, whereas could synergistically grow and reproduce with Proteobacteria (especially Comamonas, Stenotrophomonas, and Bosea) in autumn. The blooms of the small centric diatom Stephanodiscus were regularly found every year in lakes and reservoirs under low temperature conditions (Ha, Jang, \& Joo, 2003; Kang et al., 2007). Stephanodiscus was only found in spring in our study area, and was significantly positive correlated with bacterial Pseudoxanthomonas. The relationships between Navicula and bacterial communities did not appear to be one sided, Navicula constituted symbiotic relationships with Flavobacterium, Massilia, Gemmata, and Aquabacterium in spring, verifying that cell yield and growth rate of Navicula and the bacterialFlavobacterium increased when grown in mixed culture, because the organic material secreted by diatoms was absorbed by and would support the growth of bacterium. The diatom Cymatopleura had high connectivity, in addition to co-growing with Sphingobium, it also repelled each other with bacteria Pedomicrobium and green algae. The genus Amphora is one of the larger genera belonging to the family Naviculaceae and widespread including freshwater, brackish water, and marine habitats (Nagumo, 2003).Amphorawas significantly positive related with cyanobacteria and most Proteobacteria (Legionella, Hyphomicrobium, and Rhizobium ) except Rhodoferax, and negative related toChlorella and Choricystis . From these results, network analysis is potentially important for clarifying the internal mechanism of interspecies interaction and grasping the functions of microbial communities in ecological processes such as carbon, nitrogen, and phosphorus dynamics.

\section{Conclusion}

The eukaryotic phytoplankton and bacterial networks constructed in our research conformed to the properties of non-randomly connections, modular structure, and "small world". The complexity of the network was susceptible to temperature, high and low temperatures were not conducive to the stability of communities. For example, eukaryotic phytoplankton networks in spring and autumn were more complex than in summer and winter. In co-occurrence patterns, the positive interactions between bacteria and eukaryotic phytoplankton OTUs may be evidence of mutual influence (cooperation), while the negative correlations may indicate predation or competition. Through its within-module connectivity $(Z i)$ and among-module connectivity $(\mathrm{Pi})$, we identified the keystone species that can maintain the stability of microbial networks. The keystone species in bacterial networks were mainly OTUs of Proteobacteria, Planctomycetes, Bacteroidetes, Verrucomicrobia, Acidobacteria, Chloroflexi, and Firmicutes, which were defined as rare taxa; and in eukaryotic phytoplankton networks, most OTUs of Chlorophyta, Bacillariophyta, and Ochrophyta belonging to rare taxa, and only 2 OTUs of Chlorophyta were abundant taxa. With the disappearance of the 
"keystone species" in the network center, the aggregation structure will suffer major damage, which may bring huge biogeochemical consequences. The abundance of these keystone species was significantly related to many ecosystem functions and environmental changes, highlighting the key ecological role of rare species, and providing a new ecological significance for the seasonal succession patterns of bacteria and eukaryotic phytoplankton in urban aquatic ecosystems.

\section{Acknowledgements}

This study was funded by the Excellent Achievement Cultivation Project of Higher education in Shanxi (no. 2020KJ029), Shanxi Province Graduate Education Innovation Project (no. 2019SY030), and the Shanxi "1331 Project".

\section{References}

Assenov, Y., Ramirez, F., Schelhorn, S., Lengauer, T., \& Albrecht, M. (2008). Computing topological parameters of biological networks. Bioinformatics, 24 (2), 282-284. doi:10.1093/bioinformatics/btm554

Azam, F. (1998). Microbial Control of Oceanic Carbon Flux: The Plot Thickens. Science, 280 (5364), 694696. doi:10.1126/science.280.5364.694

Azam, F., \& Malfatti, F. (2007). Microbial structuring of marine ecosystems. Nature Reviews Microbiology, 5 (10), 782-791. doi:10.1038/nrmicro1747

Bolan, N. S., Baskaran, S., \& Thiagarajan, S. (1996). An evaluation of the methods of measurement of dissolved organic carbon in soils, manures, sludges, and stream water.Communications in Soil Science $\mathcal{E}^{3}$ Plant Analysis, 27 (13-14), 2723-2737. doi:10.1080/00103629609369735

Bradley, D., Emma, R. M., \& Kelly, J. J. (2013). Wastewater treatment effluent reduces the abundance and diversity of benthic bacterial communities in urban and suburban rivers. Applied \& Environmental Microbiology, 79 (6), 1897-1905. doi:10.1128/AEM.03527-12

Buchan, A., Lecleir, G. R., Gulvik, C. A., \& Gonzalez, J. M. (2014). Master recyclers: features and functions of bacteria associated with phytoplankton blooms. Nature Reviews Microbiology, 12 (10), 686-698. doi:10.1038/nrmicro3326

Bunse, C., Bertosfortis, M., Sassenhagen, I., Sildever, S., Sjoqvist, C., Godhe, A., . . L Lundholm, N. (2016). Spatio-Temporal Interdependence of Bacteria and Phytoplankton during a Baltic Sea Spring Bloom. Frontiers in microbiology, 7 , 517-517. doi:10.3389/fmicb.2016.00517

Calderer, M., Marti, V., De Pablo, J., Guivernau, M., Prenafetaboldu, F. X., \& Vinas, M. (2014). Effects of enhanced denitrification on hydrodynamics and microbial community structure in a soil column system. Chemosphere, 111 , 112-119. doi:10.1016/j.chemosphere.2014.03.033

Callieri, C., Cronberg, G., \& Stockner, J. G. (2012). Freshwater picocyanobacteria: single cells, microcolonies and colonial forms. In Whitton, B. [Ed.] Ecology of Cyanobacteria II: Their Diversity in Time and Space, 2nd edn. Springer Publishers, Dordrecht, the Netherlands, pp. 229-69.

Chen, M., Chen, F., Xing, P., Li, H., \& Wu, Q. L. (2010). Microbial eukaryotic community in response to Microcystis spp. bloom, as assessed by an enclosure experiment in Lake Taihu, China. FEMS Microbiology Ecology, 74 (1), 19-31. doi:10.1111/j.1574-6941.2010.00923.x

Deng, Y., Jiang, Y., Yang, Y., He, Z., Luo, F., \& Zhou, J. (2012). Molecular ecological network analyses.BMC Bioinformatics, 13 (1), 113-113. doi:10.1186/1471-2105-13-113

Duffy, J. E., Cardinale, B. J., Mcintyre, P. B., Thebault, E., \& Loreau, M. (2007). The functional role of biodiversity in ecosystems: incorporating trophic complexity.Ecology Letters, 10 (6), 522-538. doi:10.1111/j.14610248.2007.01037.x 
Dumbrell, A. J., Nelson, M., Helgason, T., Dytham, C., \& Fitter, A. H. (2010). Relative roles of niche and neutral processes in structuring a soil microbial community. The ISME journal, 4 (3), 337-345. doi:10.1038/ismej.2009.122

Edgar, R. C., Haas, B. J., Clemente, J. C., Christopher, Q., \& Rob, K. (2011). UCHIME improves sensitivity and speed of chimera detection. Bioinformatics, 27 (16), 2194. doi:10.1093/bioinformatics/btr381

Falkowski, P. G., Fenchel, T., \& Delong, E. F. (2008). The microbial engines that drive Earth's biogeochemical cycles. Science, 320 (5879), 1034-1039. doi:10.1126/science.1153213

Fan, K., Weisenhorn, P., Gilbert, J. A., \& Chu, H. (2018). Wheat rhizosphere harbors a less complex and more stable microbial co-occurrence pattern than bulk soil. Soil Biology and Biochemistry, 125 , 251-260. doi:10.1016/j.soilbio.2018.07.022

Gücker, B., Brauns, M., \& Pusch, M. T. (2009). Effects of wastewater treatment plant discharge on ecosystem structure and function of lowland streams. Journal of the North American Benthological Society, 25 (2), 313329. doi:10.1899/0887-3593(2006)25[313:EOWTPD]2.0.CO;2

Gücker, B., Brauns, M., Solimini, A. G., Voss, M., Walz, N., \& Pusch, M. T. (2011). Energy Agency, Vienna, Austria. Urban stressors alter the trophic basis of secondary production in an agricultural stream. Journal Canadien Des Sciences Halieutiques Et Aquatiques, 68 (1), 74-88. doi:10.1139/F10-126

Ger, K. A., Urrutiacordero, P., Frost, P. C., Hansson, L., Sarnelle, O., Wilson, A. E., \& Lurling, M. (2016). The interaction between cyanobacteria and zooplankton in a more eutrophic world. Harmful Algae, 54 , 128-144. doi:10.1016/j.hal.2015.12.005

Gregory, B., Ochsenkühn, M. A., Cong, F., Jhamal, F., Koester, J. A., \& Amin, S. A. (2018). Bacterial Communities of Diatoms Display Strong Conservation Across Strains and Time. Frontiers in microbiology, 9 , 659-. doi:10.3389/fmicb.2018.00659

Guimera, R., \& Amaral, L. A. N. (2005). Functional cartography of complex metabolic networks.Nature, 433 (7028), 895-900. doi:10.1038/nature03288

Ha, K., Jang, M., \& Joo, G. (2003). Winter Stephanodiscus bloom development in the Nakdong River regulated by an estuary dam and tributaries. Hydrobiologia , 221-227. doi:10.1023/B:HYDR.0000008564.64010.4c

Haas, B. J., Gevers, D., Earl, A. M., Feldgarden, M., Ward, D. V., Giannoukos, G., . . Sodergren, E. (2011). Chimeric 16S rRNA sequence formation and detection in Sanger and 454-pyrosequenced PCR amplicons. Genome research, 21 (3), 494-504. doi:10.1101/gr.112730.110

Herlemann, D. P. R., Labrenz, M., Jurgens, K., Bertilsson, S., Waniek, J. J., \& Andersson, A. F. (2011). Transitions in bacterial communities along the $2000 \mathrm{~km}$ salinity gradient of the Baltic Sea. The ISME journal, 5 (10), 1571-1579. doi:10.1038/ismej.2011.41

Hornerdevine, M. C., \& Bohannan, B. J. M. (2006). Phylogenetic clustering and overdispersion in bacterial communities. Ecology, 87 (7). doi:10.1890/0012-9658(2006)87[100:PCAOIB]2.0.CO;2

Hunt, D. E., \& Ward, C. S. (2015). A network-based approach to disturbance transmission through microbial interactions. Frontiers in microbiology, 6 , 1182-1182. doi:10.3389/fmicb.2015.01182

Jo, H. (2004). Metagenomics: application of genomics to uncultured microorganisms. Microbiol Mol Biol Rev, 68 (4), 669-685. doi:10.1128/MMBR.68.4.669-685.2004

Jones, A. C., Liao, T. S. V., Najar, F. Z., Roe, B. A., Hambright, K. D., \& Caron, D. A. (2013). Seasonality and disturbance: annual pattern and response of the bacterial and microbial eukaryotic assemblages in a freshwater ecosystem.Environmental Microbiology, 15 (9), 2557-2572. doi:10.1111/1462-2920.12151

Jongman, R. H. G., Ter Braak, C. F. J., \& Van Tongeren, O. F. R. (1995) Data Analysis in Community and Landscape Ecology . United Kingdom: Cambridge University Press. 
Jousset, A., Bienhold, C., Chatzinotas, A., Gallien, L., Gobet, A., Kurm, V., . . Salles, J. F. (2017). Where less may be more: how the rare biosphere pulls ecosystems strings. The ISME journal, 11 (4), 853-862. doi:10.1038/ismej.2016.174

Ju, F., Xia, Y., Guo, F., Wang, Z., \& Zhang, T. (2014). Taxonomic relatedness shapes bacterial assembly in activated sludge of globally distributed wastewater treatment plants.Environmental Microbiology, 16 (8), 2421-2432. doi:10.1111/1462-2920.12355

Kanehisa, M., Goto, S., Sato, Y., Furumichi, M., \& Tanabe, M. (2012). KEGG for integration and interpretation of large-scale molecular data sets. Nucleic Acids Research, 40, 109-114. doi:10.1093/nar/gkr988

Kang, Y. H., Kim, B. R., Choi, H. J., Seo, J. G., Kim, B. H., \& Han, M. (2007). Enhancement of algicidal activity by immobilization of algicidal bacteria antagonistic to Stephanodiscus hantzschii (Bacillariophyceae).Journal of Applied Microbiology, 103 (5), 1983-1994. doi:10.1111/j.1365-2672.2007.03439.x

Konopka, A., Lindemann, S. R., \& Fredrickson, J. K. (2015). Dynamics in microbial communities: unraveling mechanisms to identify principles. The ISME journal, 9 (7), 1488-1495. doi:10.1038/ismej.2014.251

Krienitz, L., Bock, C., Dadheech, P. K., \& Proschold, T. (2011). Taxonomic reassessment of the genus Mychonastes (Chlorophyceae, Chlorophyta) including the description of eight new species. Phycologia, 50 (1), 89-106. doi:10.2216/10-15.1

Lawrence, J. R., Swerhone, G. D. W., Wassenaar, L. I., \& Neu, T. R. (2005). Effects of selected pharmaceuticals on riverine biofilm communities. Canadian Journal of Microbiology, 51 (8), 655-669. doi:10.1139/w05-047

Lee, C. S., Oh, H., Oh, H., Kim, H., \& Ahn, C. (2016). Two-phase photoperiodic cultivation of algal-bacterial consortia for high biomass production and efficient nutrient removal from municipal wastewater. Bioresource Technology, 200 , 867-875. doi:10.1016/j.biortech.2015.11.007

Li, Y. H., Hu, M. M., Shen, Y. W., Liu, Y. D., Dun, H., \& Gen, B. (2013). Mychonastes, a new recorded genus of freshwater Chlorophyceae isolated from a Chinese lake. Acta Hydrobiologica Sinica, 37 (3), 473-480.

Liu, C., Shi, X., Fan, F., Wu, F., \& Lei, J. (2019). N:P ratio influences the competition of Microcystis with its picophytoplankton counterparts, Mychonastes and Synechococcus, under nutrient enrichment conditions. Journal of Freshwater Ecology, 34 (1), 445-454. doi:10.1080/02705060.2019.1622604

Logares, R., Lindstrom, E. S., Langenheder, S., Logue, J. B., Paterson, H., Laybournparry, J., . . Bertilsson, S. (2013). Biogeography of bacterial communities exposed to progressive long-term environmental change. The ISME journal, 7 (5), 937-948. doi:10.1038/ismej.2012.168

Ma, B., Wang, H., Dsouza, M., Lou, J., He, Y., Dai, Z., . . Gilbert, J. A. (2016). Geographic patterns of co-occurrence network topological features for soil microbiota at continental scale in eastern China. The ISME journal, 10 (8), 1891-1901. doi:10.1038/ismej.2015.261

Mallon, C. A., Poly, F., Roux, X. L., Marring, I., Van Elsas, J. D., \& Salles, J. F. (2015). Resource pulses can alleviate the biodiversity-invasion relationship in soil microbial communities. Ecology, 96 (4), 915-926. doi:10.1890/14-1001.1

Markowitz, V. M., Chen, I. A., Palaniappan, K., Chu, K., Szeto, E., Grechkin, Y., . . Williams, P. (2012). IMG: the integrated microbial genomes database and comparative analysis system. Nucleic Acids Research, $40,115-122$. doi:10.1093/nar/gkr1044

Nagumo, T. (2003). Taxonomic studies of the subgenus Amphora Cleve of-the genus Amphora (Bacillariophyceae) in Japan. Stuttgart: Science Publishers.

Park, M., Ryu, S. H., Vu, T. T., Ro, H., Yun, P., \& Jeon, C. O. (2007). Flavobacterium defluvii sp. nov., isolated from activated sludge. International Journal of Systematic and Evolutionary Microbiology, 57 (2), 233-237. doi:10.1097/01.NNA.0000295631.72721.17 
Pedrosalio, C. (2012). The Rare Bacterial Biosphere. Annual Review of Marine Science, 4 (1), 449-466. doi:10.1146/annurev-marine-120710-100948

Pester, M., Bittner, N., Deevong, P., Wagner, M., \& Loy, A. (2010). A 'rare biosphere' microorganism contributes to sulfate reduction in a peatland. The ISME journal, 4 (12), 1591-1602. doi:10.1038/ismej.2010.75

Pinhassi, J., Sala, M. M., Havskum, H., Peters, F., Guadayol, O., Malits, A., \& Marrase, C. (2004). Changes in Bacterioplankton Composition under Different Phytoplankton Regimens. Applied and Environmental Microbiology, 70 (11), 6753-6766. doi:10.1128/aem.70.11.6753-6766.2004

Prosser, J. I., Bohannan, B. J., Curtis, T. P., Ellis, R. J., Firestone, M. K., Freckleton, R. P., . . Lennon, J. J. (2007). The role of ecological theory in microbial ecology. Nature Reviews Microbiology, 5 (5), 384-392. doi:10.1038/nrmicro1643

Rastogi, G., \& Sani, R. K. (2011). Molecular techniques to assess microbial community structure, function, and dynamics in the environment. In Ahmad I, Ahmad F, Pichtel J (Eds.), Microbes and microbial technology . (pp. 29-57). New York: Springer Science.

Shi, S., Nuccio, E. E., Shi, Z. J., He, Z., Zhou, J., \& Firestone, M. K. (2016). The interconnected rhizosphere: High network complexity dominates rhizosphere assemblages.Ecology Letters, 19 (8), 926-936. doi:10.1111/ele.12630

Stanish, L. F., O’Neill, S. P., Gonzalez, A., Legg, T. M., Knelman, J., McKnight, D. M., . . . Nemergut, D. R. (2013). Bacteria and diatom co-occurrence patterns in microbial mats from polar desert streams. Environmental Microbiology, 15 (4), 1115-1131. doi:10.1111/j.1462-2920.2012.02872.x

Steele, J. A., Countway, P. D., Xia, L., Vigil, P. D., Beman, J. M., Kim, D. Y., . . . Schwalbach, M. S. (2011). Marine bacterial, archaeal and protistan association networks reveal ecological linkages. The ISME journal, 5 (9), 1414-1425. doi:10.1038/ismej.2011.24

Stegen, J. C., Lin, X., Konopka, A., \& Fredrickson, J. K. (2012). Stochastic and deterministic assembly processes in subsurface microbial communities. The ISME journal, 6 (9), 1653-1664. doi:10.1038/ismej.2012.22

Sun, Z., Li, G., Wang, C., Jing, Y., Zhu, Y., Zhang, S., \& Liu, Y. (2015). Community dynamics of prokaryotic and eukaryotic microbes in an estuary reservoir. Scientific Reports, 4 (1), 6966-6966. doi:10.1038/srep06966

Waiser, M. J., Tumber, V., \& Holm, J. (2011). Effluent-dominated streams. Part 1: Presence and effects of excess nitrogen and phosphorus in Wascana Creek, Saskatchewan, Canada.Environmental Toxicology $\mathcal{G}$ Chemistry, 30 (2), 496-507. doi:10.1002/etc.399

Wang, Q., Garrity, G. M., Tiedje, J. M., \& Cole, J. R. (2007). Naive Bayesian classifier for rapid assignment of rRNA sequences into the new bacterial taxonomy. Applied and Environmental Microbiology, 73 (16), 52615267. doi:10.1128/AEM.00062-07

Wang, Y. S., Lou, Z. P., Sun, C. C., \& Song, S. (2008). Ecological Environment Changes In Daya Bay, China, From 1982 To 2004. Marine Pollution Bulletin, 56 (11), p.1871-1879. doi:10.1016/j.marpolbul.2008.07.017

Worden, A. Z., Follows, M. J., Giovannoni, S. J., Wilken, S., Zimmerman, A. E., \& Keeling, P. J. (2015). Rethinking the marine carbon cycle: Factoring in the multifarious lifestyles of microbes. Science, 347 (6223), 735-735. doi:10.1126/science.1257594

Xue, Y., Chen, H., Yang, J. R., Liu, M., Huang, B., \& Yang, J. (2018). Distinct patterns and processes of abundant and rare eukaryotic plankton communities following a reservoir cyanobacterial bloom. The ISME journal, 12 (9), 2263-2277. doi:10.1038/s41396-018-0159-0

Yang, C., Wang, Q., Simon, P. N., Liu, J., Liu, L., Dai, X., . . . Pan, X. (2017). Distinct network interactions in particle-associated and free-living bacterial communities during a Microcystis aeruginosa bloom in a plateau lake.Frontiers in microbiology, 8 , 1202. doi:10.3389/fmicb.2017.01202 
Yannarell, A. C., \& Triplett, E. W. (2005). Geographic and environmental sources of variation in lake bacterial community composition. Applied and Environmental Microbiology, 71 (1), 227-239. doi:10.1128/AEM.71.1.227-239.2005

Yu, Z., Yang, J., Zhou, J., Yu, X., Liu, L., \& Lv, H. (2014). Water Stratification Affects the Microeukaryotic Community in a Subtropical Deep Reservoir. Journal of Eukaryotic Microbiology, 61 (2), 126-133. doi:10.1111/jeu.12090

Zhang, W., Pan, Y., Yang, J., Chen, H., Holohan, B., Vaudrey, J. M. P., . . . Mcmanus, G. B. (2018). The diversity and biogeography of abundant and rare intertidal marine microeukaryotes explained by environment and dispersal limitation.Environmental Microbiology, 20 (2), 462-476. doi:10.1111/1462-2920.13916

Zhou, J., Deng, Y., Luo, F., He, Z., Tu, Q., \& Zhi, X. (2010). Functional Molecular Ecological Networks.Mbio, 1 (4). doi:10.1128/mBio.00169-10

Zhu, C. M., Zhang, J. Y., Nawaz, M. Z., Mahboob, S., Al-Ghanim, K. A., Khan, I. A., . . . Chen, T. (2019). Seasonal succession and spatial distribution of bacterial community structure in a eutrophic freshwater Lake, Lake Taihu. Science of The Total Environment, 66. doi: 10.1016/j.scitotenv.2019.03.087

Zhu, J., Hong, Y., Zada, S., Hu, Z., \& Wang, H. (2018). Spatial Variability and Co-acclimation of Phytoplankton and Bacterioplankton Communities in the Pearl River Estuary, China. Frontiers in microbiology, 9 . doi:10.3389/fmicb.2018.02503

Ziegler, M., Eguiluz, V. M., Duarte, C. M., \& Voolstra, C. R. (2018). Rare symbionts may contribute to the resilience of coral-algal assemblages. The ISME journal, 12 (1), 161-172. doi:10.1038/ismej.2017.151

\section{Data availability statement}

The datasets generated and analyzed during the current study are available in the Sequence Read Archive (SRA) of NCBI repository, under accession number SRP273360.

\section{Authors contribution}

Jia Feng and Shulian Xie conceived and designed the experiments. Jing Yang conducted the laboratory experiments and wrote the paper. Jing Yang, Haiguang Pei, Junping Lv, Qi Liu, Fangru Nan, and Xudong Liu analyzed and interpreted the results. Jia Feng and Shulian Xie reviewed the manuscript. All authors approved the final manuscript.

Table 1 Description statistics of main physical and chemical in Fenhe River

\begin{tabular}{lllllll}
\hline Seasonal & Variables & $\mathrm{NO}_{3}{ }^{-}-\mathrm{N}$ & $\mathrm{NO}_{2}^{-}-\mathrm{N}$ & $\mathrm{PO}_{4}{ }^{3-}-\mathrm{P}$ & $\mathrm{Chl} \mathrm{a}$ & $\mathrm{DOC}$ \\
\hline \multirow{5}{*}{ Spring } & $\mathrm{Unit}$ & $\mathrm{mg} \mathrm{L}^{-1}$ & $\mathrm{mg} \mathrm{L}^{-1}$ & $\mathrm{mg} \mathrm{L}^{-1}$ & $\mathrm{mg} \mathrm{m}^{3}$ & $\mathrm{mg} \mathrm{L}^{-1}$ \\
& $\mathrm{~S} 1$ & 1.110 & 0.030 & 0.087 & 8.390 & 6.360 \\
& $\mathrm{~S} 2$ & 1.820 & 0.031 & 0.092 & 10.140 & 6.850 \\
& $\mathrm{~S} 3$ & 1.540 & 0.035 & 0.090 & 10.840 & 6.690 \\
& $\mathrm{~S} 4$ & 1.680 & 0.029 & 0.098 & 9.800 & 6.860 \\
& $\mathrm{~S} 5$ & 1.800 & 0.042 & 0.112 & 11.370 & 6.960 \\
& $\mathrm{~S} 6$ & 1.480 & 0.043 & 0.090 & 11.010 & 6.900 \\
& Average & 1.572 & 0.035 & 0.095 & 10.258 & 6.770 \\
& $\mathrm{~S} 1$ & 1.210 & 0.083 & 0.212 & 27.000 & 6.395 \\
& $\mathrm{~S} 2$ & 2.332 & 0.164 & 0.128 & 29.400 & 6.745 \\
& $\mathrm{~S} 3$ & 1.329 & 0.170 & 0.129 & 26.900 & 6.435 \\
& $\mathrm{~S} 4$ & 0.942 & 0.142 & 0.142 & 31.400 & 6.059 \\
& $\mathrm{~S} 5$ & 0.848 & 0.155 & 0.192 & 51.900 & 5.399 \\
& S6 & 0.951 & 0.165 & 0.155 & 59.400 & 6.162 \\
& Average & 1.269 & 0.147 & 0.160 & 37.667 & 6.199
\end{tabular}




\begin{tabular}{lllllll}
\hline Seasonal & Variables & $\mathrm{NO}_{3}{ }^{-}-\mathrm{N}$ & $\mathrm{NO}_{2}{ }^{-}-\mathrm{N}$ & $\mathrm{PO}_{4}{ }^{3-}-\mathrm{P}$ & $\mathrm{Chl} \mathrm{a}$ & $\mathrm{DOC}$ \\
\hline Autumn & $\mathrm{S} 1$ & 0.298 & 0.033 & 0.075 & 14.030 & 5.938 \\
& $\mathrm{~S} 2$ & 1.486 & 0.056 & 0.067 & 33.830 & 6.416 \\
& $\mathrm{~S} 3$ & 0.520 & 0.079 & 0.067 & 32.770 & 5.876 \\
& $\mathrm{~S} 4$ & 1.561 & 0.050 & 0.034 & 31.780 & 6.522 \\
& $\mathrm{~S} 5$ & 0.322 & 0.020 & 0.097 & 51.170 & 6.534 \\
& $\mathrm{~S} 6$ & 0.224 & 0.017 & 0.034 & 83.430 & 6.404 \\
& Average & 0.735 & 0.043 & 0.062 & 41.168 & 6.282 \\
Winter & $\mathrm{S} 1$ & 0.499 & 0.030 & 0.104 & 0.560 & 6.020 \\
& $\mathrm{~S} 2$ & 0.550 & 0.030 & 0.016 & 1.958 & 6.974 \\
& $\mathrm{~S} 3$ & 0.420 & 0.037 & 0.038 & 0.526 & 5.818 \\
& $\mathrm{~S} 4$ & 0.200 & 0.023 & 0.052 & 0.543 & 5.818 \\
& S5 & 0.216 & 0.051 & 0.060 & 2.257 & 5.916 \\
& S6 & 0.159 & 0.037 & 0.082 & 1.244 & 5.970 \\
& Average & 0.341 & 0.035 & 0.059 & 1.181 & 6.086 \\
\hline
\end{tabular}

\section{Figure captions:}

Fig. 1 The location of the sampling sites in Fenhe River

Fig. 2 The relative abundance of bacteria communities at the genera level

Fig. 3 The relative abundance of eukaryotic phytoplankton communities at the genera level

Fig. 4 Co-occurrence networks constructed based on bacterial communities. Each node represents an OTU. Red and green lines connecting nodes (edges) represent positive and negative relationships, respectively.

Fig. 5 Co-occurrence networks constructed based on eukaryotic phytoplankton communities. Each node represents an OTU. Red and green lines connecting nodes (edges) represent positive and negative relationships, respectively.

Fig. 6 Heatmap based on the correlation between environmental factors and predicted functional abundance of bacteria. * denotes $P<0.05,{ }^{* *}$ denotes $P<0.01$.

Fig. 7 Heatmap based on correlation between environmental factors and keystone species of eukaryotic phytoplankton. ${ }^{*}$ denotes $P<0.05$, ** denotes $P<0.01$.

Fig. 8 Seasonal co-occurrence patterns between bacteria and eukaryotic phytoplankton at the genera level 


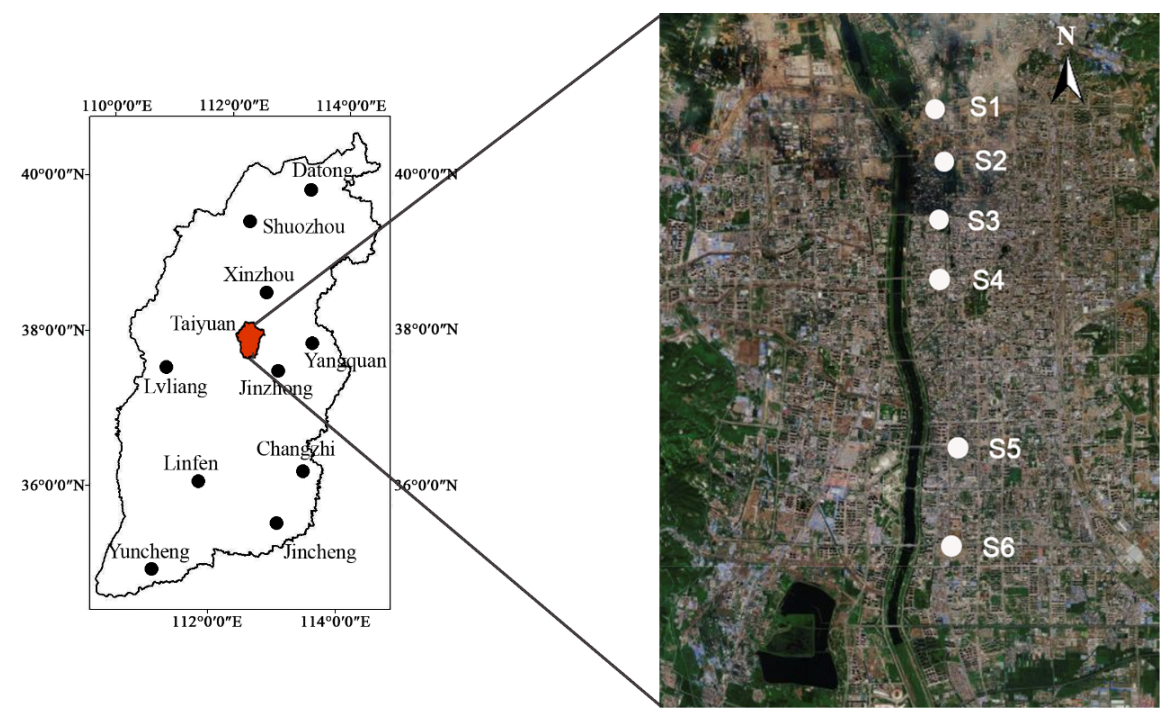

Fig. 1 The location of the sampling sites in Fenhe River
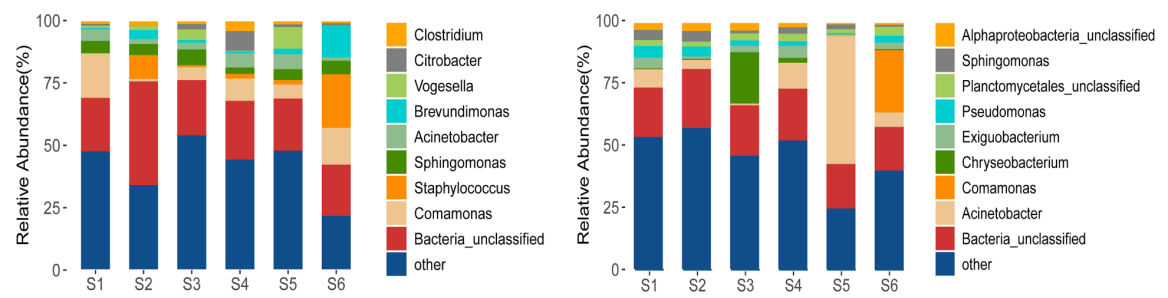

(a) Spring

(b) Summer

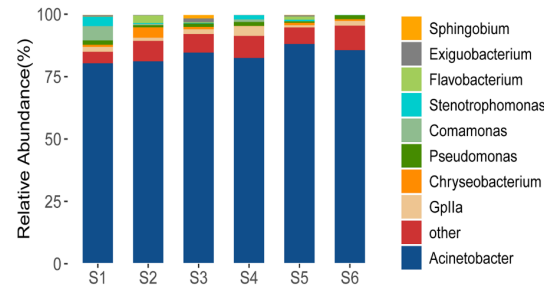

(c) Autumn

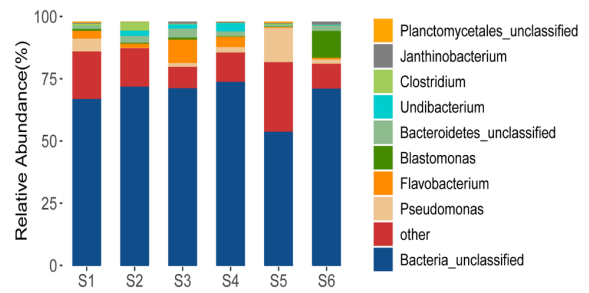

(d) Winter

Fig. 2 The relative abundance of bacteria communities at the genera level 


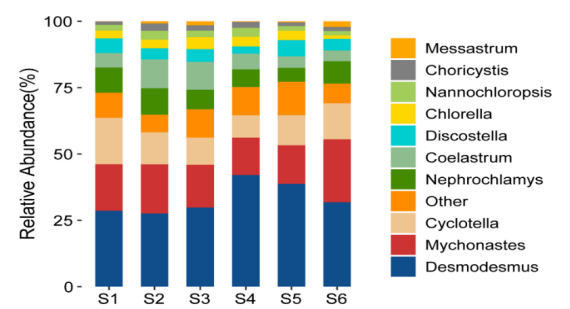

(a) Spring

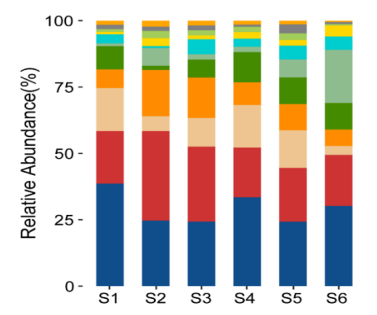

(c) Autumn

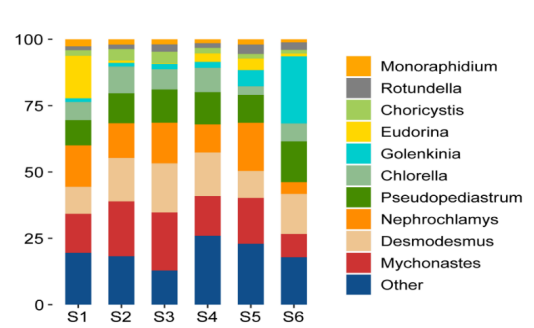

(b) Summer

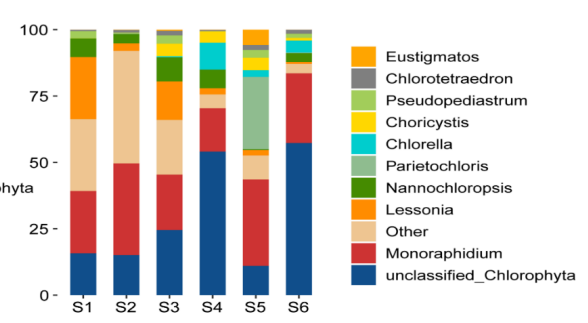

(d) Winter

Fig. 3 The relative abundance of eukaryotic phytoplankton communities at the genera level

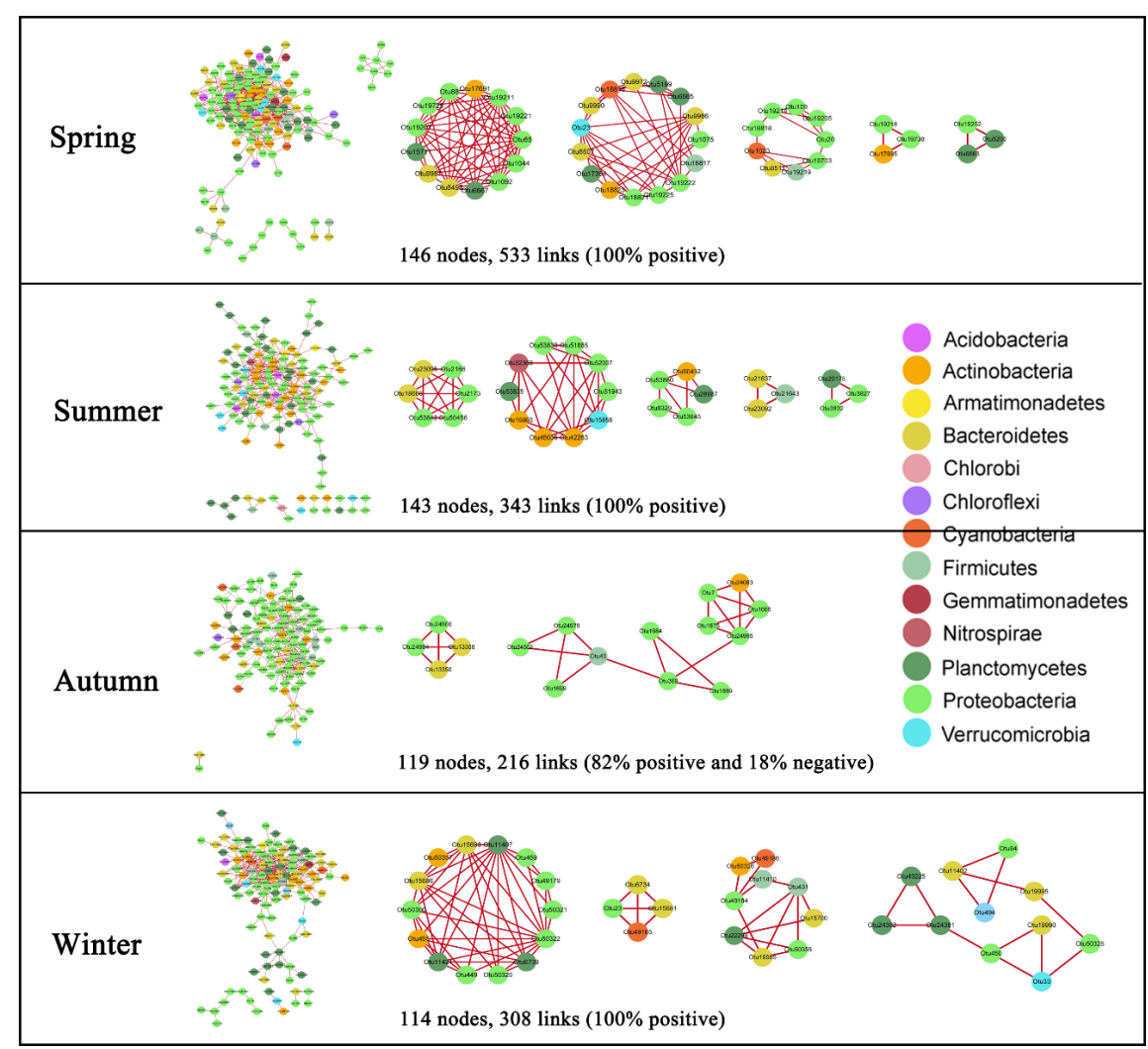

Fig. 4 Co-occurrence networks constructed based on bacterial communities. Each node represents an OTU. Red and green lines connecting nodes (edges) represent positive and negative relationships, respectively. 


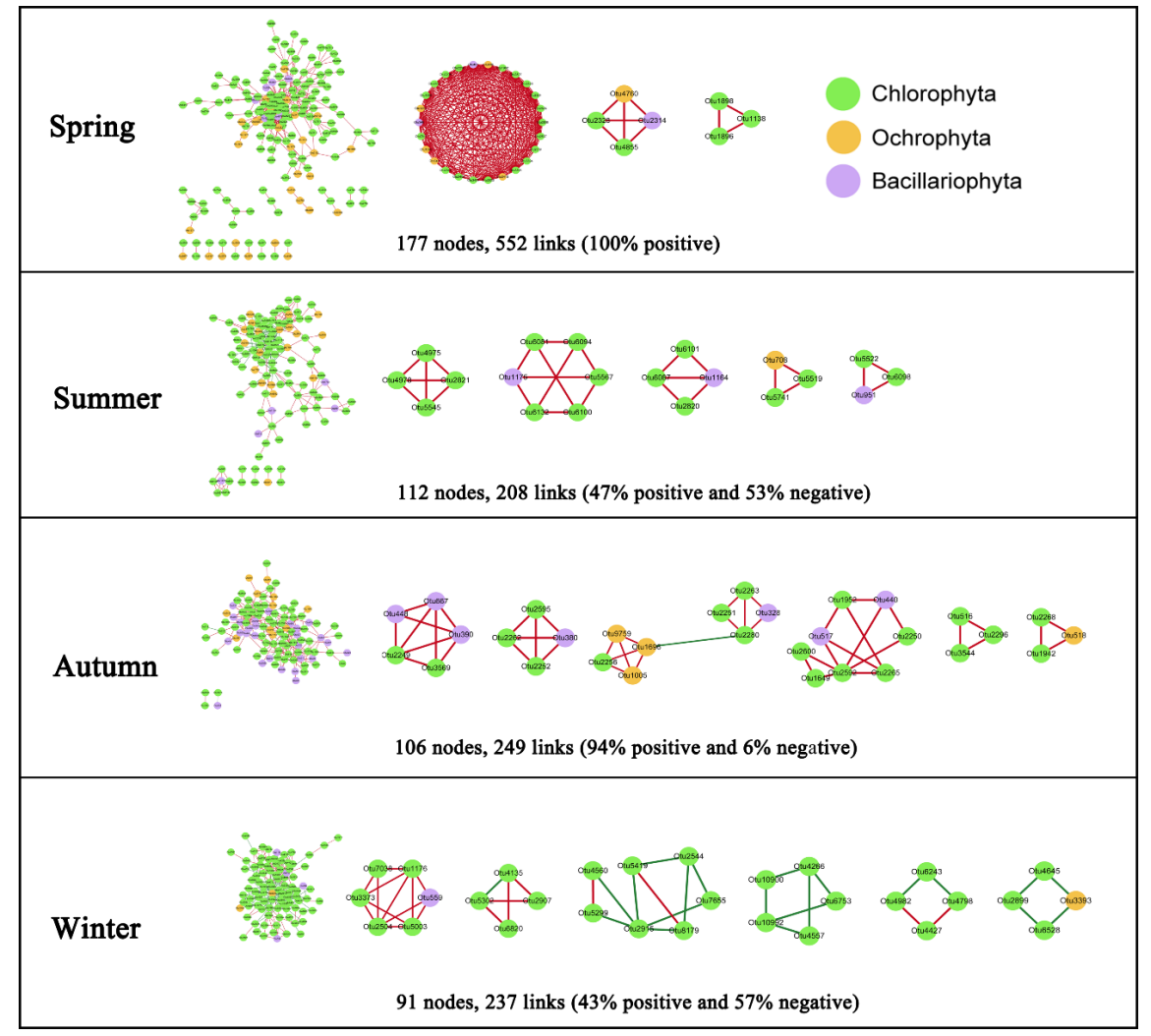

Fig. 5 Co-occurrence networks constructed based on eukaryotic phytoplankton communities. Each node represents an OTU. Red and green lines connecting nodes (edges) represent positive and negative relationships, respectively.

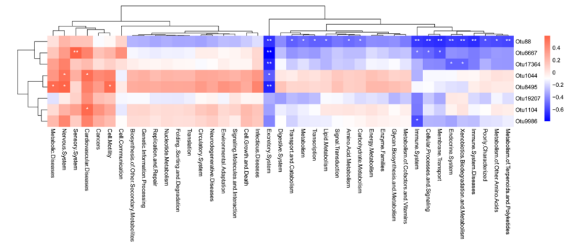

(a) Spring

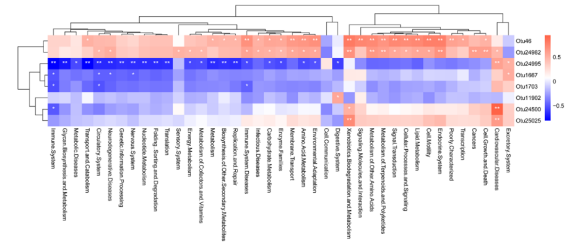

(c) Autumn

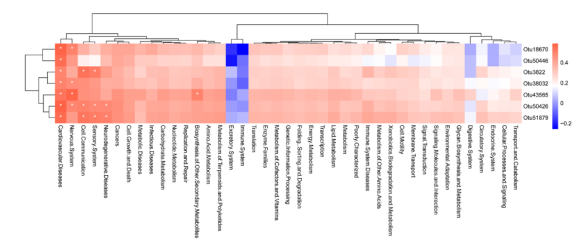

(b) Summer

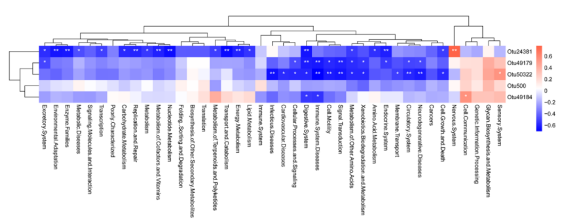

(d) Winter

Fig. 6 Heatmap based on the correlation between keystone species and predicted functional abundance of bacteria. * denotes $P<0.05$, ** denotes $P<0.01$. 


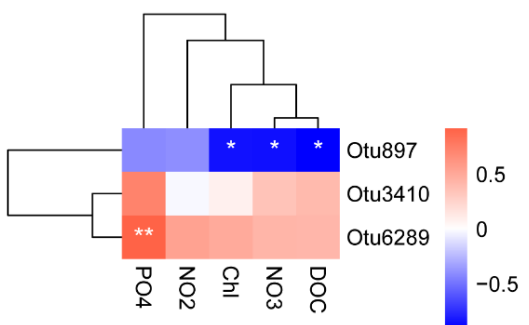

(a) Spring

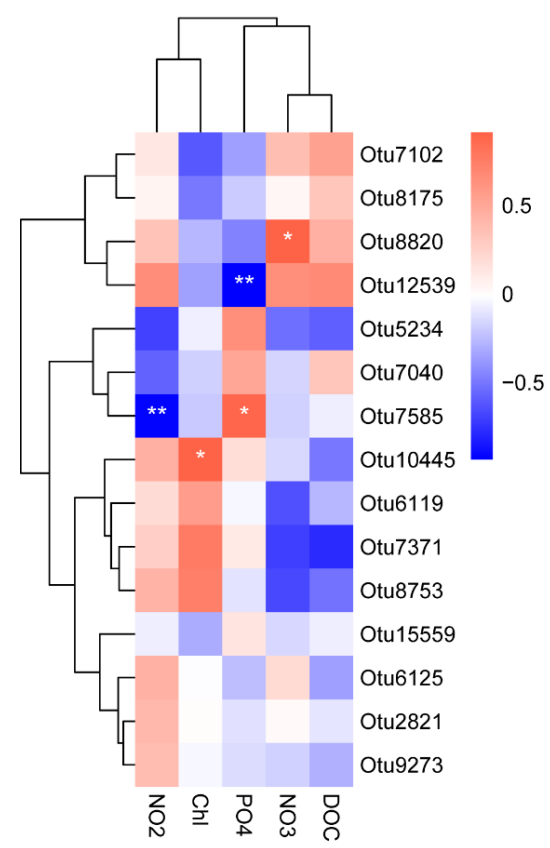

(b) Summer

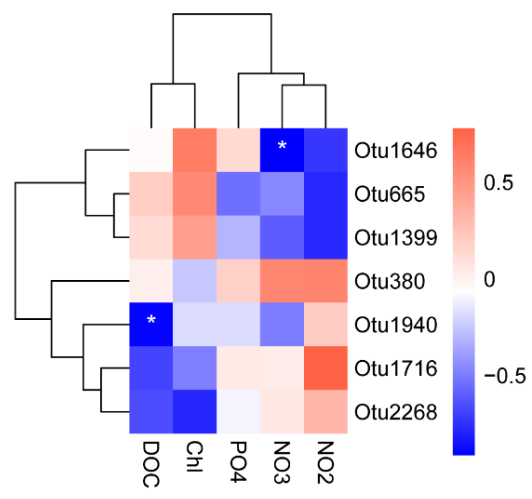

(c) Autumn

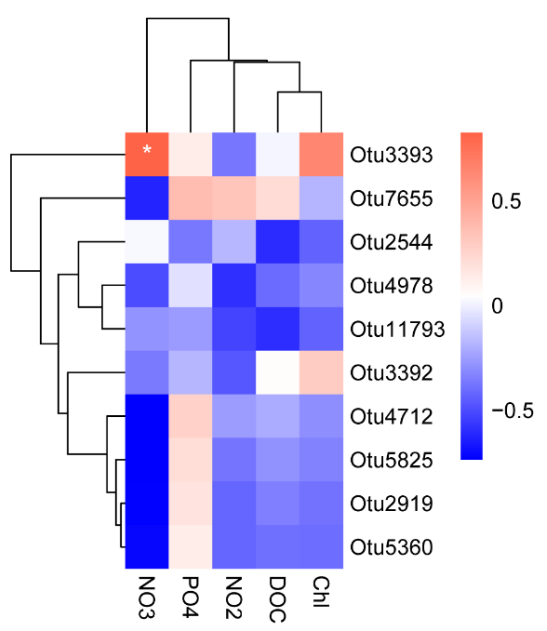

(d) Winter

Fig. 7 Heatmap based on correlation between environmental factors and keystone species of eukaryotic phytoplankton. ${ }^{*}$ denotes $P<0.05,{ }^{*}$ denotes $P<0.01$. 


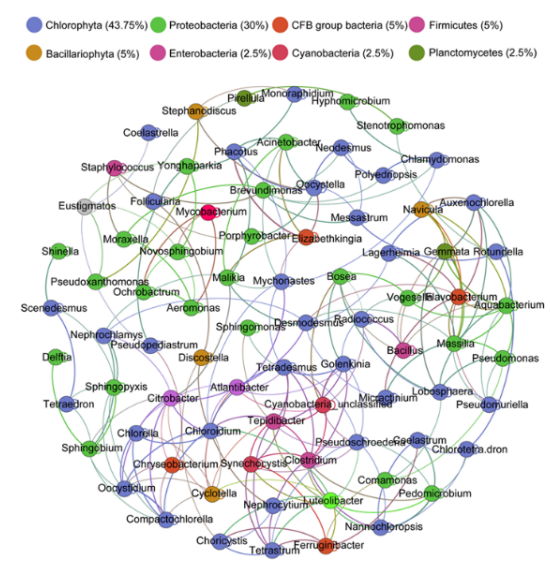

(a) Spring

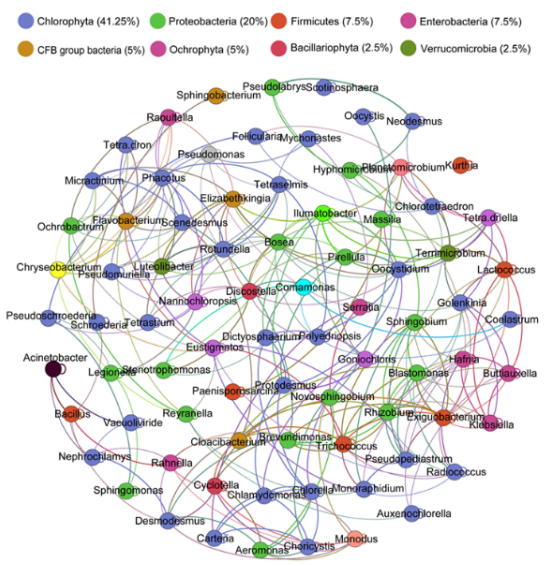

(c) Autumn

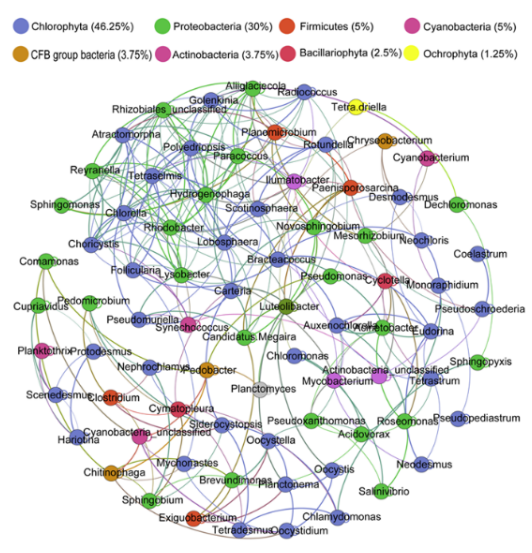

(b) Summer

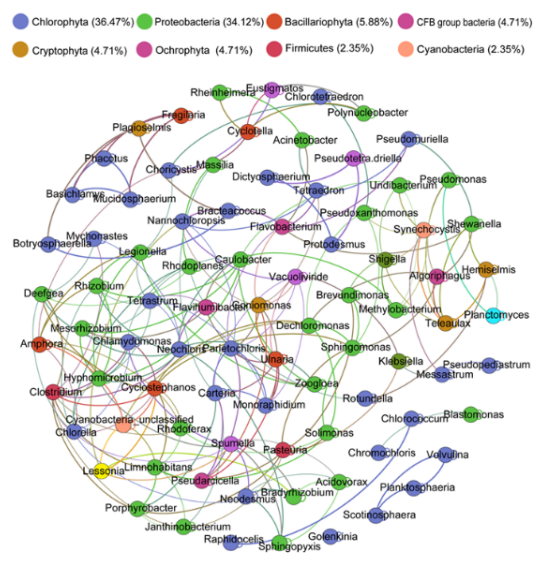

(d) Winter

Fig. 8 Seasonal co-occurrence patterns between bacteria and eukaryotic phytoplankton at the genera level 